D.O.I.: $10.3895 /$ gi.v11n3.2759

\title{
CONFIABILIDADE DE SISTEMAS REDUNDANTES COM REPARO EM UMA REFINARIA DE PETRÓLEO
}

\section{RELIABILITY OF REDUNDANT SYSTEMS WITH REPAIR IN AN OIL REFINERY}

\author{
Lucas Silveira Santos ${ }^{1}$; Fernanda Pinotti ${ }^{2}$; José Luis Duarte Ribeiro ${ }^{3}$; Heitor Cabral Botelho ${ }^{4}$ \\ ${ }^{1}$ Universidade Federal do Rio Grande do Sul - Brasil \\ silveira.santos@ufrgs.br \\ ${ }^{2}$ Universidade Federal do Rio Grande do Sul - Brasil \\ fernandapinotti06@gmail.com \\ ${ }^{3}$ Universidade Federal do Rio Grande do Sul - Brasil \\ ribeiro@producao.ufrgs.br \\ ${ }^{4}$ Petrobrás \\ hcbotelho@petrobras.com.br
}

\begin{abstract}
Resumo
Sistemas com alta confiabilidade são, atualmente, imprescindiveis para as empresas, por questões de produtividade, disponibilidade e segurança. A redundância dos equipamentos é uma das formas utilizadas para assegurar tal necessidade, exigindo, paralelamente, análises quantitativas das falhas resultantes das condições operacionais às quais os equipamentos estão submetidos. Este estudo foi motivado pela necessidade de uma refinaria de petróleo avaliar e aumentar a confiabilidade de seus sistemas redundantes. Assim, este artigo apresenta uma análise quantitativa da confiabilidade dos sistemas e propõe alterações para aumentar a confiabilidade de tais sistemas. Trata-se de um estudo de caso que se baseia em análises quantitativas, contemplando a modelagem de distribuições de probabilidade e curvas de sensibilidade, para avaliar as falhas nos equipamentos e a confiabilidade dos sistemas. A partir dos resultados, foi possível perceber a forte relação existente entre a confiabilidade dos sistemas, o tipo de arranjo dos equipamentos e os respectivos tempos de reparo e de bom funcionamento dos componentes.
\end{abstract}

Palavras-chave: manutenção; confiabilidade; sistemas com redundância; indústria petroquímica.

\section{Introdução}

Tem-se obervado que empresas dos diversos segmentos do mercado estão sendo exigidas a inovarem em seus processos e alcançarem níveis ótimos de qualidade e produtividade para permanecerem competitivas. No contexto atual, as pressões mercadológicas impostas por esse cenário de expressiva competitividade implicam a intolerância a perdas decorrentes de falhas em equipamentos, diante do comprometimento que essas podem representar em termos de lucratividade para as organizações. Por conta disso, exige-se que a manutenção seja vista como uma função 
estratégica das empresas (MENDES, 2011), que pode contribuir para maximizar a disponibilidade dos equipamentos, minimizar custos operacionais e aumentar a produtividade e segurança (KARDEC; RIBEIRO, 2002).

Uma parcela significativa das empresas ainda não tem aproveitado técnicas preditivas associadas à manutenção preventiva para atuarem em suas plantas industriais. O setor de manutenção ainda tem atuado de forma reativa, por meio de manutenções mal planejadas ou até mesmo com a aplicação preponderante de ações corretivas (DESHPAND; MODAK, 2001), nas quais o reparo do equipamento é feito após a ocorrência da falha, o que resulta no aumento dos gastos e das perdas produtivas.

Segundo Ribeiro e Fogliatto (2009), tem-se percebido uma forte relação entre manutenção e confiabilidade, já que a primeira é realizada no intuito de reduzir falhas ou restaurar o estado operante do sistema. Em outras palavras, a manutenção visa manter e melhorar a confiabilidade de operação do sistema de produção, o que justifica a implementação de programas de manutenção centrados em confiabilidade, principalmente em indústrias nas quais acidentes com potenciais perdas materiais e humanas são condicionados por falhas em equipamentos. Nesse sentido, a gestão da manutenção torna-se complexa, principalmente em se tratando de empresas do ramo petroquímico.

Em virtude da necessidade das empresas incorporarem abordagens quantitativas na gestão da manutenção, que podem contribuir para melhorar o desempenho nessa área, e considerando que a literatura carece deste tipo de abordagem (MENDES, 2011), este artigo tem como principal objetivo realizar estudos quantitativos de confiabilidade em uma refinaria de petróleo localizada na região metropolitana de Porto Alegre.

Diante das exigências relativas à segurança e à produtividade, a refinaria em estudo possui sistemas com redundância de equipamentos críticos, no intuito de garantir a disponibilidade dos mesmos. A empresa busca entender de que modo é possível aumentar a confiabilidade dos sistemas implantados. Assim configura-se a oportunidade de atuação, cujos objetivos são avaliar quantitativamente a confiabilidade dos esquemas de redundância utilizados, por meio da avaliação dos índices Mean Time Between Failures (MTBF) e Mean Time to Repair (MTTR) e dos seus efeitos sobre as curvas de confiabilidade dos sistemas, além de propor ajustes que possam aumentar a confiabilidade mediante a alteração do arranjo ou das práticas de manutenção preventiva e corretiva.

O presente estudo se justifica, portanto, pela importância do tema abordado, particularmente no ambiente industrial estudado. O tema é relevante, pois a manutenção de equipamentos é a forma pela qual as empresas buscam evitar falhas e paradas de produção, que possuem forte impacto no gerenciamento produtivo (SLACK et Al, 2002). No que diz respeito ao ambiente estudado, Bignetti 
(2008) afirma que os desafios e as preocupações referentes aos reparos e paradas no âmbito de indústrias do ramo petroquímico são explicados pela alta tecnologia envolvida, pelas grandes escalas de produção, pelo processo dinâmico e contínuo e pelo processamento de um bem de alto valor agregado.

O estudo de caso limita-se a explorar métodos quantitativos de análise da confiabilidade dos sistemas, assumindo-se ainda que as falhas dos equipamentos analisados seguem uma distribuição exponencial, dado que foram estudados os sistemas redundantes sujeitos a manutenção preventiva e reparos. Além disso, é considerado que a troca dos equipamentos é feita instantaneamente quando um deles falha, garantindo, desta maneira, a redundância do sistema. Ademais, não foram considerados dados de custos e de investimentos que poderiam ser avaliados concomitantemente com as simulações envolvendo diferentes arranjos dos sistemas, o que traria uma avaliação econômica interessante para o trabalho, sendo sugerido como um estudo a ser explorado.

Este artigo está dividido em cinco seções. Esta, que contempla a Introdução, apresentando o problema de pesquisa e seus objetivos. Após, uma seção referente ao Referencial Teórico que embasa o trabalho realizado, seguida da Metodologia empregada. As seções 4 e 5 apresentam, respectivamente, os resultados obtidos e as conclusões que consolidam o estudo.

\section{Referencial Teórico}

Confiabilidade é definida por Fogliatto e Ribeiro (2009) como a probabilidade de um componente ou sistema manter seu desempenho adequado durante um período de tempo especificado e submetido a condições ambientais predeterminadas. Por esse motivo, conhecer a confiabilidade dos sistemas ou equipamentos é de extrema relevância em relação à manutenção e, consequentemente, à qualidade.

Manutenção é definida como um conjunto de atividades que existem a fim de evitar a degradação dos equipamentos e das instalações, causada pelo seu desgaste natural e pelo uso (XENOS, 2004, p.18). A forte relação envolvendo manutenção e confiabilidade é explicada, portanto, pelo objetivo da primeira em manter e melhorar as condições operacionais do sistema produtivo. Nesse sentido, é necessário entender, também, os conceitos relativos à Manutenção Corretiva e à Manutenção Preventiva. Segundo Cavalca (2000), ações corretivas visam restaurar um item após a sua falha a uma condição especificada, ou seja, nada é feito até a ocorrência das falhas, sendo necessário o reparo ou a substituição imediata do equipamento. A manutenção preventiva envolve ações periódicas no sentido de manter a condição especificada de operação, por meio de inspeções sistemáticas, lubrificações, entre outras ações de prevenção à falha. 
Sendo a confiabilidade uma função probabilística, é necessária a utilização de distribuições de probabilidade para que se possa fazer um estudo quantitativo. As mais utilizadas em estudos de confiabilidade são: normal, exponencial, lognormal e Weibull. (CROWE; FEINBERG, 2001).

A utilização da distribuição exponencial em estudos de confiabilidade é bastante difundida (HENZ, 1997), principalmente pela simplicidade matemática associada a ela. As Equações 1, 2 e 3 determinam as representações de confiabilidade para $t \geq 0$, sendo $f(t)$ a função de densidade, $R(t) a$ função de confiabilidade e $\mathrm{h}(\mathrm{t})$ a função de risco, onde $\lambda$ é a taxa de falha. $\mathrm{O}$ cálculo para $\lambda$, por sua vez, é representado pela equação 4, onde $n$ é o número unidades em teste, $r$ é o número de unidades que falharam, $t i$ é o tempo até a falha e $t i^{+}$o tempo de censura da unidade $i$ (FOGLIATTO; RIBEIRO, 2009).

$$
\begin{aligned}
& f(t)=\lambda e^{\lambda t} \\
& R(t)=e^{-\lambda t} \\
& h(t)=\lambda \\
& \lambda=\frac{r}{\sum_{i=1}^{y} t i+\sum_{i=1}^{n-y} t i^{+}}
\end{aligned}
$$

Uma importante propriedade da distribuição exponencial apresentada por Fogliatto e Ribeiro (2009) determina que, em qualquer período do tempo, é considerada a mesma confiabilidade. Essa premissa restringe a utilização da distribuição exponencial quando a ocorrência de falhas for relativamente constante, como é o caso de unidades durante seu período de vida útil. Práticas de manutenção preventiva em certas situações resultam em equipamentos com taxas de falhas constantes ao longo do tempo, uma vez que as condições de operação se mantêm renovadas, o que implica a adequação dos tempos até a falha a um modelo de distribuição exponencial.

Ainda no âmbito do cálculo da confiabilidade de componentes ou sistemas, há alguns parâmetros que devem ser conhecidos. Segundo Ribeiro (2014), a confiabilidade pode ser avaliada de uma maneira alternativa utilizando o Tempo Médio entre Falhas (MTBF) aplicado a produtos que podem ser reparados, como é o caso de equipamentos industriais. O autor cita ainda a importância de avaliar quantitativamente a Manutenabilidade, normalmente utilizando o Tempo Médio de Reparo (MTTR). As Equações 5 e 6 representam, respectivamente, o cálculo para MTBF e MTTR quando se trabalha com a distribuição exponencial em estudos de confiabilidade, onde tr é o tempo de reparo e $n r$ o número de reparos. (RIBEIRO, 2014). 


$$
\begin{aligned}
& \text { MTBF }=\frac{\sum_{\mathrm{i}=1}^{r} t i+\sum_{\mathrm{i}=1}^{n-r} t i^{+}}{r}=\frac{1}{\lambda} \\
& \text { MTTR }=\frac{\sum t r}{n r}
\end{aligned}
$$

O termo redundância é utilizado quando mais de um equipamento estão disponíveis para a realização de uma mesma função (MENDES, 2011), por isso sistemas que utilizam redundâncias se tornam mais confiáveis à medida que diminui a probabilidade de falha durante certo período de tempo. Esses sistemas são amplamente utilizados nas indústrias, nas quais processos de risco exigem altos níveis de confiabilidade. Por conta disso, tais sistemas são encontrados, por exemplo, em refinarias de petróleo, indústrias de aço, indústrias aeronáuticas e indústrias nucleares. Os tipos de redundância se diferenciam por características específicas dos sistemas de que fazem parte. Nesse sentido, os itens redundantes podem estar ativos ou em standby.

A redundância ativa é caracterizada por um sistema no qual todos os itens estão em operação e o sistema não deixa de funcionar quando um ou mais itens falham. A redundância ativa total é aquela em que o sistema falha somente quando todos os itens que o constituem falham. Para a redundância ativa parcial o número de equipamentos que pode falhar é inferior ao caso da redundância ativa total, já que, para o sistema continuar operando, mais de um item precisa estar funcionando. No caso da redundância ativa condicional, o funcionamento do sistema é condicionado pelo modo de falha de seus itens constituintes, ou seja, se dois ou mais itens falharem no mesmo modo de falha, o sistema para. Por outro lado, se a falha ocorrer de modo diferente entre os dois, o sistema continuará funcionando (SMITH, 2001).

No caso da redundância standby, são utilizados itens adicionais ou de reserva no sistema, sendo acionados apenas quando o item em operação e que apresenta a mesma função para de funcionar (SMITH, 2001). Para o funcionamento de tais sistemas, são necessários sensores responsáveis pela detecção de falhas e pela troca dos itens.

A Figura 1 mostra que para sistemas redundantes a confiabilidade é maior do que para sistemas sem redundância no início da vida útil, tendo um declínio abrupto em determinado momento. Além disso, percebe-se que quanto maior é o número de itens redundantes, maior é o período com elevadas confiabilidade (SMITH, 2001). 
Figura 1 - Relação entre a quantidade de itens redundantes e a taxa de falhas e confiabilidade dos sistemas
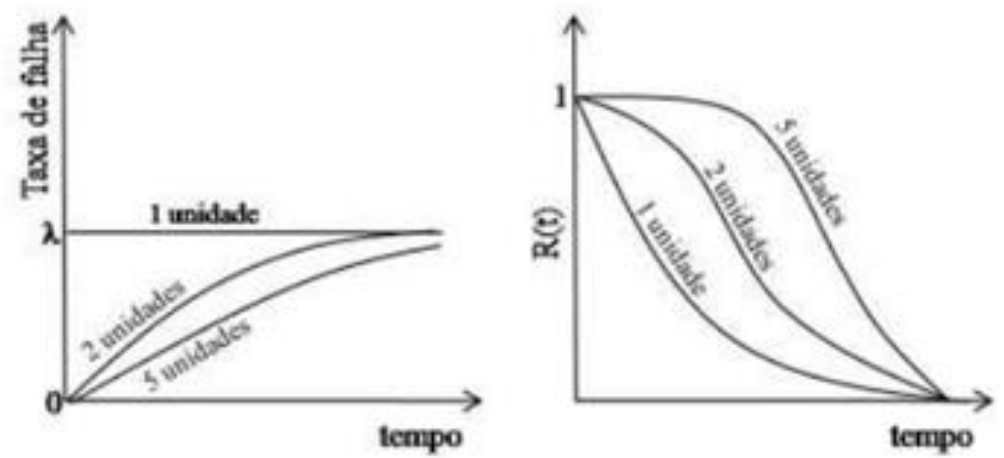

Fonte: adaptado de Smith (2001)

Ao utilizar redundância em sistemas, pode-se observar uma melhora na confiabilidade, sendo essa maior para sistemas com redundância standby do que com redundância ativa. Isso se justifica pelo fato de que os itens que constituem o sistema standby operam por menos tempo, suas falhas são independentes (não causam ou aceleram a falha dos demais). Na Figura 2, essa comparação é evidenciada para o caso particular de taxas de falhas constantes (distribuição exponencial), sendo facilmente percebido que sistemas redundantes apresentam menor taxa de falha e maior confiabilidade do que sistemas sem redundância, e que a redundância em standby possui maior confiabilidade e menor taxa de falha na comparação com a redundância ativa (SMITH, 2001).

Figura 2 - Efeito do tipo de redundância empregada na taxa de falha e na confiabilidade do sistema
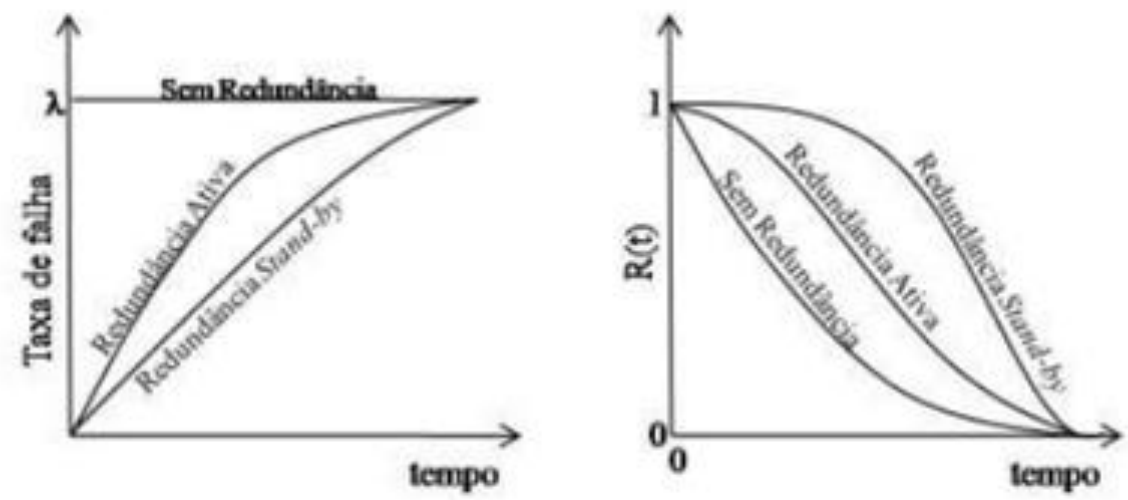

Fonte: adaptado de Smith (2001)

A forma pela qual os sistemas redundantes são monitorados também é importante ao se realizar estudos de confiabilidade. Nesses sistemas, quando não é possível monitorar continuamente, inspeções periódicas são necessárias para assegurar que o sistema esteja operando adequadamente e que o componente redundante esteja pronto para operar quando for solicitado. Essas inspeções, cujos intervalos são predeterminados, permitem detectar falhas escondidas em componentes e repará-las. Nesse sentido, deve-se encontrar o intervalo ótimo entre inspeções para 
maximizar a disponibilidade e a segurança e minimizar os custos (LAPA et al., 2006). Inspeções frequentes aumentam a disponibilidade, mas também envolvem maiores custos relativos à manutenção preventiva. Por outro lado, longos períodos entre inspeções diminuem os custos de inspeção, mas aumentam os custos de manutenção corretiva, já que há intervalos de tempo maiores nos quais o sistema pode estar inoperante (GRAY, 1985; FLAMMINI et al., 2009).

\section{Metodologia}

Este trabalho teve como proposta realizar a modelagem de confiabilidade de sistemas redundantes importantes para a planta industrial em estudo e que apresentam diferentes arranjos. A partir da análise do cenário atual, que possibilitou caracterizar os diferentes arranjos redundantes, foi possível estudar alternativas para aumento de capacidade ou de confiabilidade. As etapas executadas para a realização do estudo de caso estão apresentadas na sequência:

a) Entendimento in loco dos sistemas: um parque industrial de um processo contínuo e dinâmico que caracteriza as indústrias petroquímicas é composto por equipamentos distintos, com funções variadas e que processam produtos de alto valor agregado. No intuito de se avaliarem os sistemas mais apropriados para serem objetos do estudo, foi realizada uma visita in loco guiada por um gerente de manutenção da empresa. Através da visita realizada ao longo da área operacional da refinaria, foi possível compreender o funcionamento dos equipamentos da área de interesse, os modos de falhas recorrentes, as práticas de manutenção corretiva, preditiva e preventiva, as características dos sistemas com redundância - tipo de arranjo dos equipamentos, tipo de redundância (ativa ou passiva), envolvendo entrada automática ou manual no caso de falha;

b) Priorização dos sistemas observados: a partir do panorama observado, foram estabelecidos, juntamente com o gerente de manutenção, critérios para a escolha dos sistemas a serem estudados, dentre eles: grau de criticidade dos equipamentos para o restante da planta, sistemas redundantes com arranjos distintos, número de registros de falhas associadas ao sistema e banco de dados de falha completo, que permitisse obter o tempo real em operação dos equipamentos a serem estudados;

c) Coleta e tratamento dos dados: em um estudo de confiabilidade é imprescindível que se tenham dados confiáveis, consolidados e bem estruturados. As empresas utilizam softwares que dão suporte à gestão da manutenção, armazenando e integrando os dados de falhas de equipamentos (MACHADO, 2013). Nesse sentido, foram selecionados os dados de interesse para a realização do estudo de confiabilidade, dentre eles os registros de falhas e os tempos de reparo dos equipamentos;

d) Cálculo de MTBF e MTTR: após a etapa de coleta e tratamento dos dados, foram obtidos os intervalos de bom funcionamento (TBFs), subtraindo a data de falha da data que ele começou a 
operar, sempre considerando o tempo que o equipamento esteve efetivamente operando neste intervalo. Esse procedimento foi realizado para todos os dados de falhas dos equipamentos que compunham cada um dos sistemas de interesse. Para o cálculo do $M T B F$, foram agrupados os intervalos de bom funcionamento dos equipamentos de cada um dos sistemas, já que as falhas dos componentes pertencentes a um mesmo sistema ocorrem devido às condições de processo que os mesmos estão submetidos. Em razão disso, é esperado que a taxa de falha seja similar para os componentes de um mesmo sistema. Por fim, foram obtidos os tempos de reparo (TRs), a partir da data da falha e da data do retorno da manutenção, para então calcular o MTTR para cada um dos sistemas;

e) Modelagem de confiabilidade: a fim de modelar a confiabilidade dos sistemas, foi preciso analisar os dados de falhas para determinar a qual distribuição probabilística eles melhor se ajustavam. Nessa análise, com auxílio do software ProConf, foram realizados testes de aderência. Após isso, modelou-se a confiabilidade dos sistemas de acordo com as equações referentes à distribuição escolhida;

f) Estudo de alternativas para aumento da capacidade e/ou confiabilidade: uma vez obtidas as curvas de confiabilidade para cada um dos sistemas atuais da empresa, buscou-se avaliar o impacto da aquisição de um novo componente nas curvas de confiabilidade dos sistemas. Para tanto, foram testados diferentes arranjos, um deles priorizando o aumento de capacidade, e o outro priorizando o aumento de confiabilidade;

g) Análise de sensibilidade da confiabilidade a melhorias no MTBF e/ou MTTR: para cada um dos arranjos com redundância estudados, foi realizada uma análise de sensibilidade. A análise tinha como objetivo evidenciar o impacto que melhorias no MTBF e/ou no MTTR geram na confiabilidade dos sistemas. Essa análise pode auxiliar a empresa na decisão de investimentos em melhorias visando aumentar o MTBF ou reduzir o MTTR.

\section{Resultados e discussão}

Os procedimentos metodológicos descritos na seção anterior fundamentaram a estratégia necessária para que se atingissem os objetivos do estudo. A apresentação dos dados e dos resultados obtidos foi dividida em uma sequência lógica e encadeada no intuito de melhor organizá-los.

\subsection{Entendimento dos sistemas e tratamento dos dados}

Tendo em vista os critérios necessários para priorizar os sistemas em um estudo de confiabilidade, descritos na Seção 3 deste artigo, a decisão tomada compreendeu a escolha de dois sistemas constituídos por bombas: o primeiro caracterizado pela presença de uma bomba em operação e uma em standby (1op1s), e o segundo cujo funcionamento envolvia duas bombas em 
operação e uma bomba em reserva (2op1s). Em ambos os sistemas, a entrada da bomba reserva ocorria de modo automático e as redundâncias eram passivas, ou seja, a bomba reserva permanecia fora de operação enquanto as outras não falhavam.

A utilização de um software de armazenamento de dados possibilitou que fossem coletados dados de falha de Janeiro de 2000 a Dezembro de 2013. Os dados coletados foram extraídos do software e transferidos para planilhas eletrônicas, a fim de possibilitar a manipulação dos mesmos. Para tratamento dos dados, foram selecionados apenas os registros que causavam a parada do equipamento, o que representava um registro de falta de condições adequadas para o funcionamento (falha). Paralelamente a isso, foram solicitados dados de variáveis relacionadas ao funcionamento dos equipamentos, para saber quando eles estavam realmente operando. Isso era necessário pelo fato de a empresa utilizar a prática de rodízio dos equipamentos em funcionamento, o que envolve paradas do equipamento mesmo que esse não tenha falhado. A variável que permitiu obter o tempo real em operação das bombas foi a amperagem, cujos registros eram armazenados também em software.

\subsection{Cálculos de $M T B F, M T T R$ e confiabilidade dos sistemas}

Ao inserir os dados de falhas no software ProConf, foi evidenciada, por meio dos testes de aderência, a adequação da distribuição exponencial aos dados de estudo. O ajuste dos dados coletados a tal distribuição era esperado, uma vez que os equipamentos de interesse são submetidos a manutenções periódicas, resultando em uma taxa de falha que se mantém aproximadamente constante ao longo do tempo. A partir disso, foi possível calcular o tempo médio entre falhas $(M T B F)$, tempo médio de reparo $(M T T R)$ e a taxa de falha $(\lambda)$ para cada um dos sistemas através das equações utilizadas quando se trabalha com a distribuição exponencial, explicitadas na Seção 2 deste artigo. Os registros de falhas das bombas foram agrupados para aquelas que constituíam um mesmo sistema, assim como os tempos de bom funcionamento e os tempos de reparo. Os valores reais (em anos) obtidos para os componentes de cada um dos sistemas em estudo estão apresentados na Tabela 1.

Tabela 1 - Parâmetros do cálculo de confiabilidade para os diferentes arranjos

\begin{tabular}{cccc}
\hline Sistema & $\boldsymbol{\lambda}$ & MTBF (anos) & MTTR (anos) \\
\hline 1op1s & 2,976 & 0,336 & 0,047 \\
2op1s & 1,105 & 0,905 & 0,041 \\
\hline
\end{tabular}

Fonte: Autoria própria (2014)

A consolidação dos índices de taxa de falha, MTBF e MTTR é importante para a modelagem de confiabilidade, já que esses são parâmetros necessários para realizar o cálculo de confiabilidade dos sistemas que seguem a distribuição exponencial. Além deles, considera-se também o intervalo 
entre inspeções como um parâmetro importante, uma vez que o equipamento pode falhar sem que isso seja percebido imediatamente, podendo haver, portanto, um gap entre o momento em que ocorreu a falha e a sua descoberta. Para o caso das bombas em estudo, o intervalo entre inspeções é nulo, uma vez que o sistema está em monitoramento contínuo, e a troca da bomba que falhou pela bomba em standby ocorre instantaneamente.

A partir da consolidação dos parâmetros, procedeu-se o cálculo da confiabilidade dos sistemas. A Equação 7 apresenta o cálculo utilizado para obter o valor da confiabilidade do sistema em cada instante de tempo. Para estabelecer tal fórmula, foi utilizada a lógica empregada em cálculos de confiabilidade utilizando distribuições exponenciais, sendo $n f(t)$ a aproximação utilizada para estimar o número de falhas esperadas para os componentes ativos em um determinado tempo $t$. Este número é calculado como o quociente entre o tempo transcorrido e o $M T B F$, ou seja, para um tempo $t$, é esperado que o componente ativo falhe $t / M T B F$ vezes. Por conseguinte, para o caso em que se tem $k$ componentes operando simultaneamente, como é o caso do sistema 2:1, onde $k=2$, o número de falhas esperadas para os componentes ativos é $k$ vezes o número de falhas esperadas para um deles, conforme mostra a Equação 8.

$$
\begin{aligned}
& R s(t)=\left(e^{- \text {IXMTTR }}\right)^{n f(t)} \\
& n f(t)=k\left(\frac{t}{M T B F}\right)
\end{aligned}
$$

As Figuras 3 e 4 mostram as curvas de confiabilidade resultantes para cada um dos sistemas estudas, as quais foram obtidas utilizando as Equações 7 e 8.

Figura 3 - Curva de confiabilidade para o arranjo 1op1s

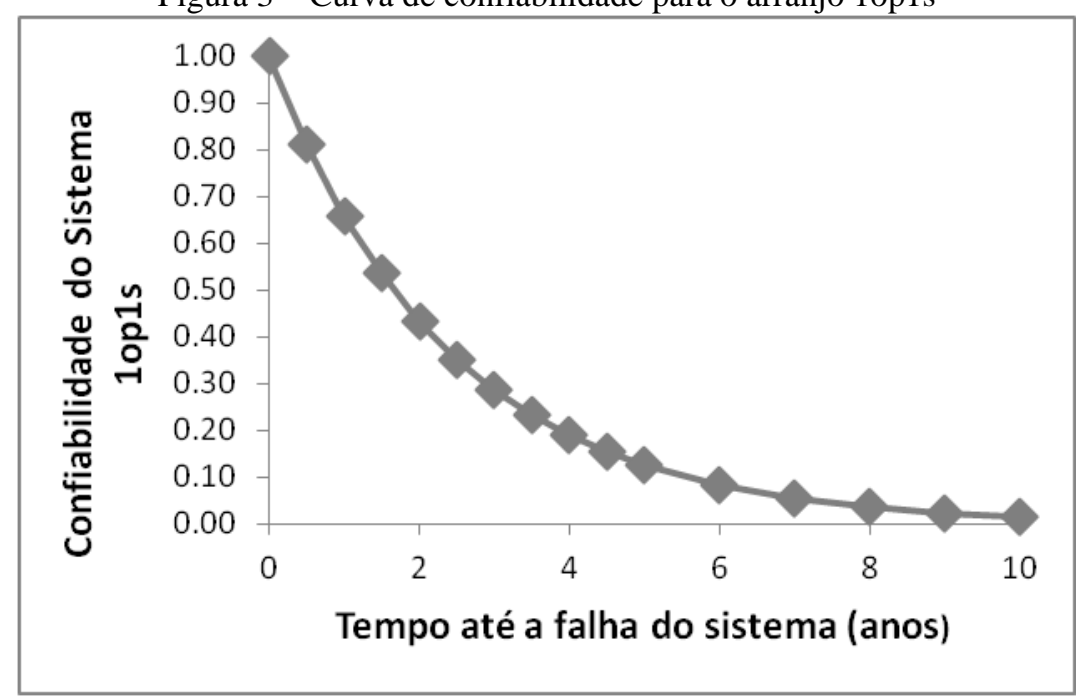

Fonte: Autoria própria (2014) 
Figura 4 - Curva de confiabilidade para o arranjo 2op1s

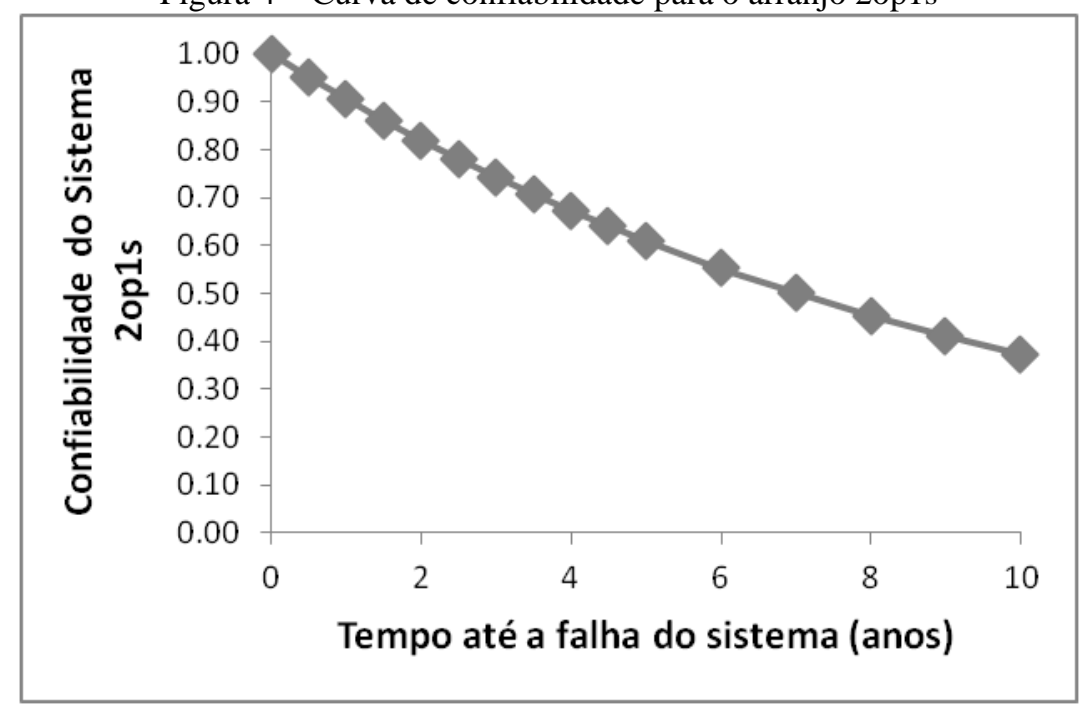

Fonte: Autoria própria (2014)

Pode-se observar através das Figuras 3 e 4 que o sistema 2op1s apresenta maior confiabilidade do que o sistema 1op1s, mesmo possuindo um componente a mais em operação, o que implica o número de falhas esperadas a cada período ser o dobro em relação ao outro sistema. Isso se explica pelo fato do MTBF do sistema 2op1s ser substancialmente maior e o MTTR menor na comparação com o sistema 1op1s, como pode ser visto na Tabela 1 apresentada anteriormente. Além disso, a curva de confiabilidade para o sistema com dois componentes ativos apresenta um declínio menos abrupto, pelas mesmas razões elencadas.

\subsection{Estudo de alternativas}

Após caracterizar os sistemas atuais da empresa, obtendo parâmetros importantes, como o $M T B F, M T T R$, taxa de falha e as curvas de confiabilidade resultantes, foi realizado um estudo referente ao impacto da aquisição de uma nova bomba para cada um dos sistemas. Com a aquisição do novo componente, pode-se priorizar um aumento da capacidade do sistema, colocando mais uma bomba em operação, ou proporcionar uma melhora na confiabilidade do sistema, mantendo a capacidade original e deixando a nova bomba em standby. Uma vez que essas alterações modificam os arranjos com redundância, é necessário modelar a confiabilidade de cada um dos novos arranjos e compará-los com a situação atual dos sistemas estudados para, então, tomar uma decisão.

Para o sistema 1op1s, a aquisição da nova bomba tendo como prioridade o aumento de confiabilidade resultou em um sistema 1op2s, enquanto que o cenário envolvendo a priorização de aumento de capacidade transformou o sistema original em um sistema 2op1s. Analogamente, para o sistema 2op1s, estudou-se a alternativa de um sistema 2op2s e de um 3op1s, priorizando, respectivamente, confiabilidade e capacidade. Para modelar a confiabilidade dos novos arranjos com redundância, foi utilizada a mesma lógica de cálculo dos sistemas atuais, explicitados 
anteriormente. Contudo, para os arranjos com mais de um componente em standby, deve-se considerar, além das falhas esperadas para o componente ativo, também o número de falhas esperadas para o primeiro componente em standby. Isso se deve ao fato de que a probabilidade do sistema falhar depende do tempo que o segundo componente em standby está em funcionamento, o que, por sua vez, depende do número de falhas do primeiro componente em standby. Assim, o número de falhas esperadas para o primeiro componente em standby, $n f 2(t)$, é estimado utilizando a aproximação apresentada na Equação 9, enquanto o cálculo de confiabilidade do sistema, $R s(t)$, para esses casos é descrito pela Equação 10.

$$
\begin{aligned}
& n f 2(t)=\frac{n f(t) \times M T T R}{M T B F} \\
& R s(t)=\left(e^{-(\lambda \times M T T R)}\right)^{n f 2(t)}
\end{aligned}
$$

As curvas de confiabilidade para as alternativas de aumento de confiabilidade ou capacidade dos sistemas estudados são apresentadas nas Figuras 5 e 6 . No caso do sistema atual 1op1s, foram analisadas as curvas de confiabilidade para 10 anos, porque na alternativa que considera aumento de capacidade a confiabilidade decresce significativamente, não havendo motivos para fazer uma análise por um período maior. Contudo, no caso do sistema atual 2op1s, as curvas de confiabilidade mantêm valores relevantes até 20 anos, justificando a escolha de diferentes escalas para os gráficos.

Figura 5 - Estudo de capacidade e confiabilidade para o arranjo 1op1s

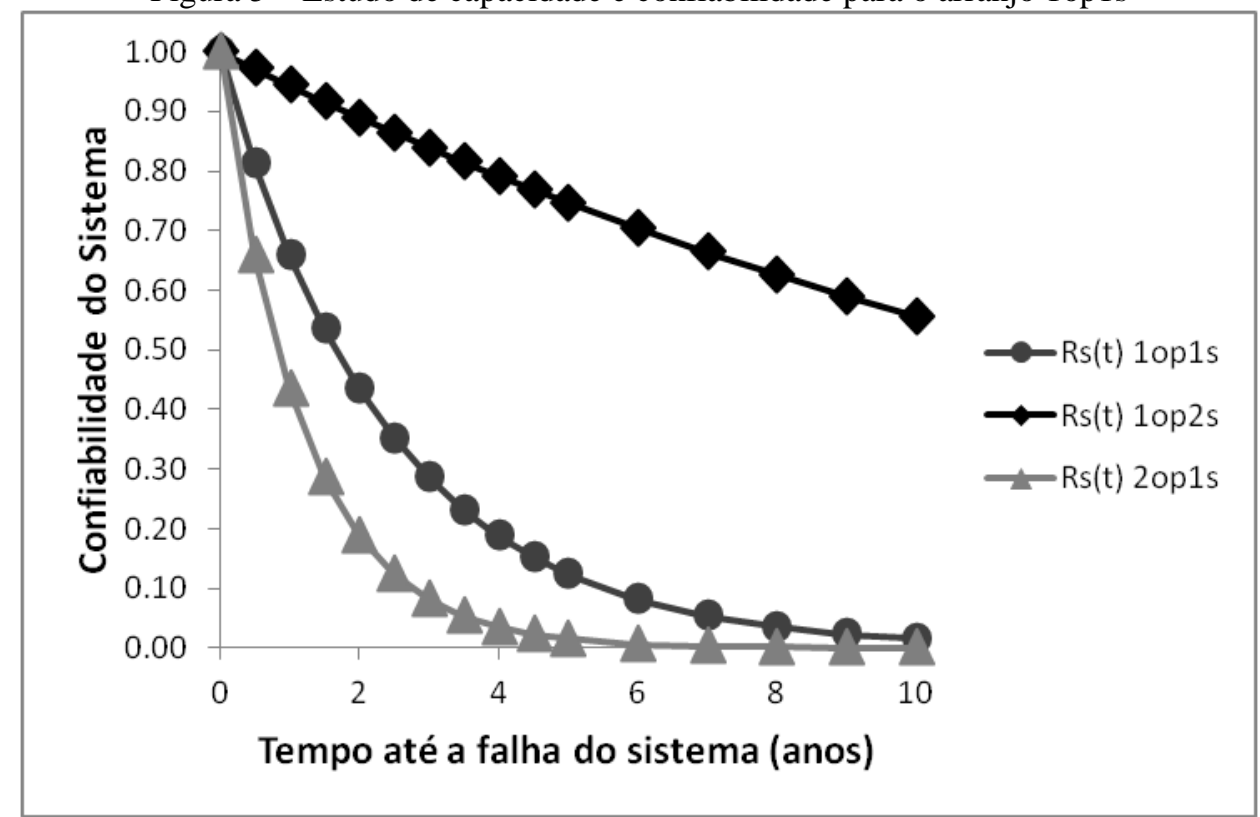

Fonte: Autoria própria (2014) 
Figura 6 - Estudo de capacidade e confiabilidade para o arranjo 2op1s

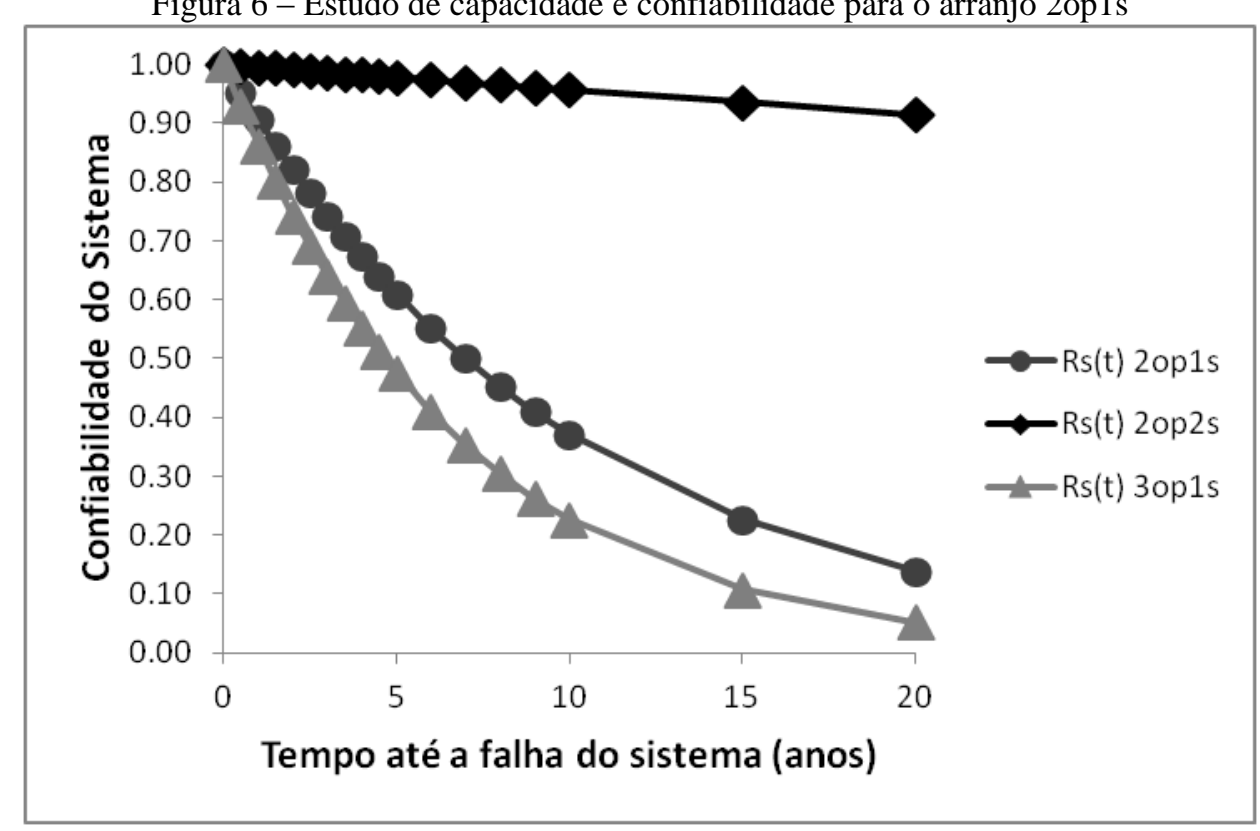

Fonte: Autoria própria (2014)

Pode-se observar que, para ambos os casos, a confiabilidade diminuiu razoavelmente ao colocar uma nova bomba em operação. Por outro lado, mantendo essa bomba adicional em standby, a confiabilidade melhora consideravelmente. A exemplo disso, para o caso da escolha do arranjo 2op2s alternativamente ao sistema atual 2op1s, conforme mostra a Figura 6, o sistema passa a apresentar aproximadamente $90 \%$ de confiabilidade para 20 anos de operação. O mesmo ocorre para o sistema 1op1s, que passa, ao priorizar a confiabilidade com a aquisição da nova bomba, a aproximadamente 55\% de confiabilidade para 10 anos, contrapondo-se a situação inicial de aproximadamente $1 \%$ para o mesmo intervalo de tempo, conforme mostra a Figura 5.

Para facilitar a comparação das alternativas, foram elaboradas as Tabelas 2 e 3, nas quais são apresentadas comparações entre os índices de confiabilidade para os três diferentes arranjos de cada um dos sistemas estudados, bem como a capacidade de cada um desses arranjos. No caso do arranjo 2op1s a análise foi feita para 10 anos, porque nesse período a confiabilidade ainda se mantém em níveis consideráveis, porém o mesmo não acontece com o arranjo 1op1s. Neste caso, optou-se por fazer a análise para 3 anos, uma vez que nesse período a confiabilidade ainda é considerável.

Tabela 2 - Comparativo entre confiabilidade e capacidade para as diferentes alternativas ao arranjo 1op1s

\begin{tabular}{cccc}
\hline & Arranjo atual (1op1s) & 1op2s & 2op1s \\
\hline Confiabilidade para 3 anos & $22,58 \%$ & $83,89 \%$ & $8,17 \%$ \\
Capacidade & 1 & 1 & 2 \\
\hline
\end{tabular}

Fonte: Autoria própria (2014)

Tabela 3 - Comparativo entre confiabilidade e capacidade para as diferentes alternativas ao arranjo 2op1s

\begin{tabular}{cccc}
\hline & Arranjo atual (2op1s) & 2op2s & 3op1s \\
\hline Confiabilidade para 10 anos & $37,15 \%$ & $95,66 \%$ & $22,65 \%$ \\
Capacidade & 2 & 2 & 3 \\
\hline
\end{tabular}

Fonte: Autoria própria (2014) 
Cabe à empresa, a partir dos gráficos e dos resultados obtidos, avaliar suas necessidades de capacidade e confiabilidade, para optar entre uma alternativa ou outra para cada um dos sistemas estudados. Esse estudo oferece amplas informações aos tomadores de decisão da empresa, permitindo maior acurácia na decisão tomada.

\subsection{Análise de sensibilidade}

A análise de sensibilidade foi realizada com o objetivo de determinar quais os parâmetros que promovem maior impacto na confiabilidade dos sistemas estudados. Para isso, foram criadas três curvas de confiabilidade a partir de melhorias em MTBF e/ou MTTR. Uma das curvas considerava a multiplicação do $M T B F$, produzindo cinco pontos igualmente espaçados, começando com o valor atual do MTBF e tendo como último ponto o dobro desse valor. Para o MTTR foi utilizada a mesma lógica, porém dividindo o valor atual do MTTR até a metade desse valor. A última curva é uma combinação das duas primeiras e, por esse motivo, é aquela que gera maior incremento na confiabilidade de ambos os sistemas, como é possível observar nas Figuras 7 e 8 :

Figura 7 - Sensibilidade da confiabilidade do sistema a melhorias em MTBF e/ou MTTR para o arranjo 1op1s

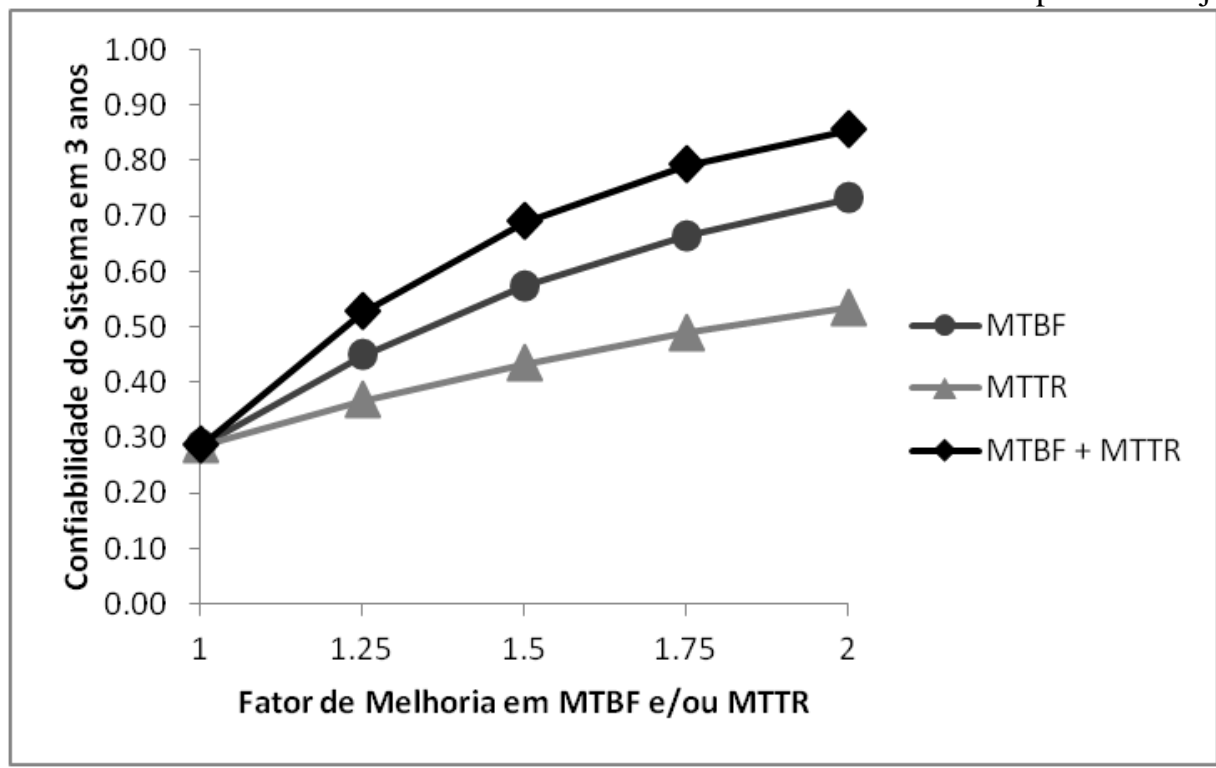

Fonte: Autoria própria (2014) 
Figura 8 - Sensibilidade da confiabilidade do sistema a melhorias em MTBF e/ou MTTR para o arranjo 2op1s

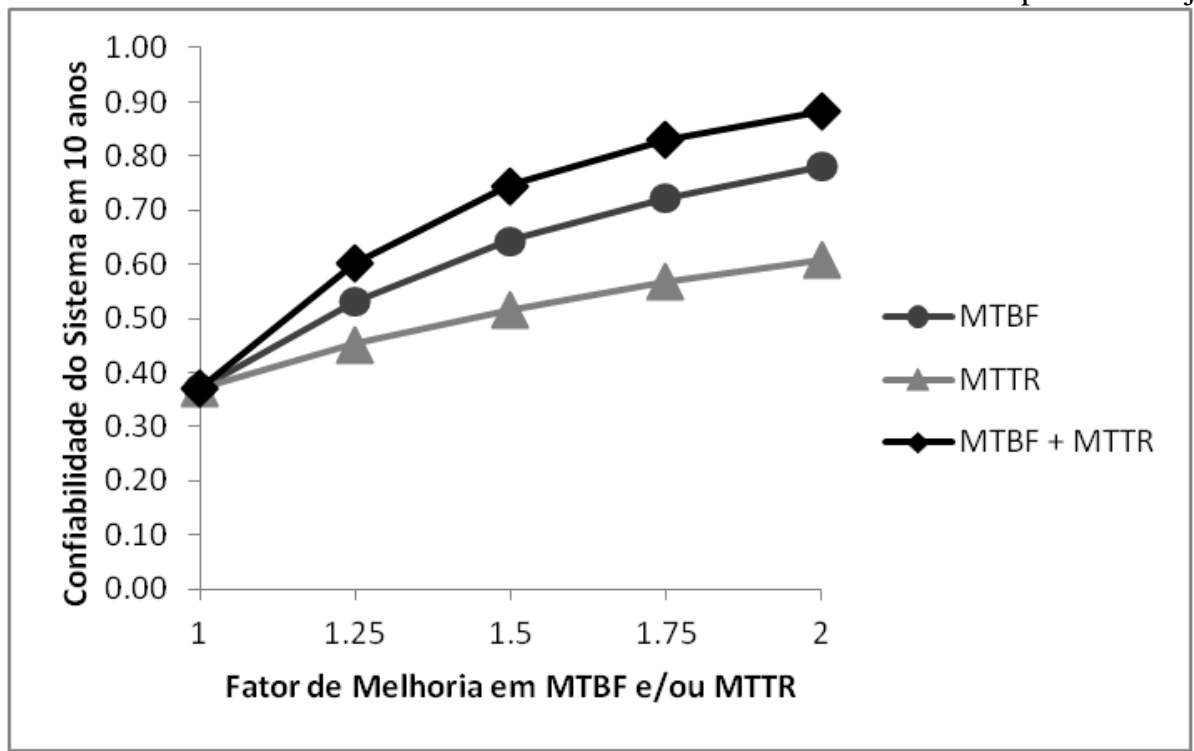

Fonte: Autoria própria (2014)

A análise de sensibilidade revela que a confiabilidade é mais sensível a melhorias em $M T B F$, o que reduz o número de falhas, do que a melhorias no $M T T R$, o que reduz o tempo de reparo, em virtude do cálculo de confiabilidade advindo da distribuição exponencial.

Para complementar o estudo de sensibilidade, foi realizada uma Simulação de Monte Carlo no intuito de se obter o tempo que os sistemas atuais da empresa ficam parados em função de quebra tanto dos componentes ativos como de seus backups. Para tanto, foram gerados tempos aleatórios até a falha e tempos aleatórios de reparo que seguissem as respectivas distribuições exponenciais observadas em campo. A partir disso, foi possível simular o número de falhas do sistema e o tempo de parada do mesmo para vinte mil observações de falhas de componentes ativos, obtendo, proporcionalmente, o tempo acumulado de parada e o número de falhas do sistema em um horizonte de três anos, para o sistema 1op1s, e em dez anos para o sistema 2op1s. Análoga e complementarmente, foi observado o impacto de 50\% de melhoria em MTBF e em MTTR no tempo de parada para cada um dos sistemas atuais da empresa. Os resultados gerados estão apresentados nas Tabelas 4 e 5.

Tabela 4 - Resultados obtidos a partir da Simulação de Monte Carlo para o arranjo 1op1s

\begin{tabular}{cccccc}
\hline & $\begin{array}{c}\text { Número de } \\
\text { paradas em 3 anos }\end{array}$ & MTBF & MTTR & $\begin{array}{c}\text { Tempo de } \\
\text { parada } \\
\text { (anos) em 3 } \\
\text { anos }\end{array}$ & $\begin{array}{c}\text { Tempo de } \\
\text { parada (dias) } \\
\text { em 3 anos }\end{array}$ \\
\hline Parâmetros atuais & 1,1 & 0,336 & 0,047 & 0,052 & 18,9 \\
Melhoria de 50\% no MTBF & 0,52 & 0,504 & 0,047 & 0,024 & 8,9 \\
Melhoria de 50\% no MTTR & 0,77 & 0,336 & 0,031 & 0,024 & 8,9 \\
Melhoria de 50\% em ambos & 0,34 & 0,504 & 0,031 & 0,011 & 3,9 \\
\hline
\end{tabular}

Fonte: Autoria própria (2014) 
Tabela 5 - Resultados obtidos a partir da Simulação de Monte Carlo para o arranjo 2op1s

\begin{tabular}{cccccc}
\hline & $\begin{array}{c}\text { Número de } \\
\text { paradas em 10 } \\
\text { anos }\end{array}$ & MTBF & MTTR & $\begin{array}{c}\text { Tempo de } \\
\text { parada } \\
\text { (anos) em 10 } \\
\text { anos }\end{array}$ & $\begin{array}{c}\text { Tempo de } \\
\text { parada (dias) } \\
\text { em 10 anos }\end{array}$ \\
\hline Parâmetros atuais & 0,97 & 0,905 & 0,040 & 0,039 & 14,2 \\
Melhoria de 50\% no MTBF & 0,44 & 0,358 & 0,040 & 0,018 & 6,4 \\
Melhoria de 50\% no MTTR & 0,64 & 0,905 & 0,027 & 0,018 & 6,4 \\
Melhoria de 50\% em ambos & 0,30 & 0,358 & 0,027 & 0,008 & 3,0 \\
\hline
\end{tabular}

Fonte: Autoria própria (2014)

De acordo com os resultados evidenciados, pode-se observar que melhorando em $50 \%$ o $M T B F$ ou o MTTR, o tempo de parada do sistema reduz-se à aproximadamente metade do valor atual, o que comprova que eles geram um mesmo impacto em termos financeiros decorrente da melhoria no tempo de parada do sistema. Por outro lado, se ações de melhorias forem destinadas à maximização do $M T B F$ e minimização do MTTR simultaneamente, melhorando em $50 \%$ ambos os índices, o tempo de parada da linha poderá ser reduzido em aproximadamente $80 \%$, o que pode representar uma redução nos custos de perda de oportunidade e, consequentemente, um ganho importante para a empresa em termos econômicos e financeiros.

\section{Conclusão}

Este artigo teve como objetivo central realizar análises quantitativas de confiabilidade considerando dois tipos de sistemas redundantes que a refinaria em estudo possui. Nesse sentido, os índices Mean Time Between Failures (MTBF) e Mean Time to Repair (MTTR) atuais dos componentes envolvidos foram avaliados e, então, foram obtidas e analisadas as curvas de confiabilidade dos arranjos com redundância, propondo ajustes que pudessem aumentar a confiabilidade.

O estudo de literatura indicou que para fazer uma análise quantitativa de confiabilidade era necessário utilizar distribuições de probabilidade, priorizando aquelas que melhor se ajustavam aos dados de falhas em estudo. A partir da adequação da distribuição exponencial aos dados de falha dos equipamentos estudados, o referencial teórico embasou os cálculos utilizados ao longo do trabalho por meio de equações conhecidas na literatura. Além disso, foi possível utilizar os conceitos de $M T B F, M T T R$ e os tipos de redundância existentes para fazer uma análise correta da situação atual referente aos arranjos com redundância e propor alterações que aumentassem a confiabilidade ou a capacidade dos sistemas em estudo.

Foram estudados dois arranjos compostos por bombas, em função da sua importância para a refinaria. Assim, foram coletados os registros de falha dos componentes desses sistemas, calculados os índices de MTBF e MTTR, para então serem modeladas as confiabilidades de cada um dos sistemas e elaboradas as análises complementares. 
Adicionalmente, foi realizado um estudo de alternativas para aumentar a confiabilidade ou a capacidade dos sistemas atuais, mediante a aquisição de uma nova bomba para cada um dos sistemas, sendo que a mesma poderia ser colocada como componente ativo (em operação) ou como standby. Ao colocar a nova bomba em operação, priorizando, assim, o aumento de capacidade, a confiabilidade do sistema diminui consideravelmente, ao passo que deixando a nova bomba em standby e mantendo a capacidade atual do sistema, a confiabilidade aumenta de forma importante. Logo, a empresa deve consultar suas necessidades de capacidade e de confiabilidade para tomar a melhor decisão no momento de alocar uma nova bomba, definindo o tipo de arranjo adequado para os seus equipamentos.

Por fim, para complementar as análises supracitadas, o estudo de sensibilidade da confiabilidade a melhorias em $M T B F$ e $M T T R$ mostrou-se importante para revelar a relação de tais índices com a confiabilidade dos sistemas. Por meio de gráficos, foi apresentada a confiabilidade de cada um dos sistemas face a diferentes percentuais de melhorias no tempo médio de reparo e no tempo médio de bom funcionamento. Este estudo evidenciou que para ambos os sistemas a confiabilidade é mais sensível a melhorias em $M T B F$, o que reduz o número de falhas, do que a melhorias em MTTR, o que diminui o tempo de reparo. Entretanto, ao realizar a Simulação de Monte Carlo, foi revelado que o tempo de parada dos sistemas sofre o mesmo percentual de redução quando são realizadas melhorias, na mesma proporção, em MTBF ou em MTTR. Por conseguinte, o ganho em termos financeiros e econômicos decorrente da redução do tempo de parada do sistema é o mesmo ao priorizar a melhoria em um ou em outro índice. Esse resultado é uma característica particular advinda do comportamento exponencial observado tanto nos dados de falhas como nos tempos de reparo dos componentes.

Sugere-se, como trabalhos futuros, a realização de análises de investimentos e de análises de custos concomitantemente às análises de confiabilidade elaboradas, a fim de fornecer ao tomador de decisão informações ainda mais completas, permitindo mais confiança na decisão tomada, seja na definição do tipo de arranjo dos sistemas, na aquisição de novos equipamentos, na promoção de ações de melhorias em MTBF ou em MTTR, entre outras. 


\begin{abstract}
Nowadays, high reliability systems are essential for companies in terms of productivity, availability and security. The equipment redundancy is used to attend these needs, but it also requires quantitative analyses of failures caused by operating conditions of equipment. This work was motivated by the need of an oil refinery to evaluate and improve the reliability of its redundant systems. A quantitative analysis of systems reliability was carried out and suggestions for improvement of system reliability are presented. It is a case study based on quantitative analysis, comprising probability distribution models and sensitivity curves to evaluate equipment failures and system reliability. The results revealed the strong connection among system reliability, type of reliability arrangement and components' repair time and time between failures.
\end{abstract}

Key-words: maintenance; reliability; systems with redundancy; petrochemical industry.

\title{
Referências
}

BIGNETTI, B. O Planejamento de uma parada de manutenção na indústria petroquímica: uma análise de ferramentas aplicadas. 2008. Curso de Especialização em Gestão Empresarial - Universidade Federal do Rio Grande do Sul.

CAVALCA, K. Apostila da Disciplina Confiabilidade em Engenharia. UNICAMP, 2000.

CROWE, D.; FEINBERG, A. Design for reliability. Boca Raton: CRC Press, 2001. 220 p. crossref

DESHPANDE, V. S.; MODAK, J. P. Application of RCM to a medium scale industry. Reliability Engineering \& System Safety, v. 77, 2002.

FLAMMINI F.; MARRONE S.; MAZZOCCA N.; VITTORINI V. A new modeling approach to the safety evaluation of $\mathbf{N}$-modular redundant computer systems in presence of imperfect maintenance. Reliability Engineering and System Safety 2009, 94:1422-32. cross ref

FOGLIATTO, F. S.; RIBEIRO, J. L. D. Confiabilidade e Manutenção Industrial. São Paulo: Campus - Elsevier, 2009.

GRAY, J. N. P. Continuous-time Markov Models in the solution of practical reliability problems. Reliability Engineering and System Safety 1985, 11(4):233-52. crossref

HENZ, L. Metodologia para caracterização do envelhecimento de equipamentos. 1997. Dissertação (Mestrado em Engenharia) - Universidade Federal de Santa Catarina, Brasil.

KARDEC, A; RIBEIRO, H. Gestão Estratégica e Manutenção Autônoma. Rio de Janeiro: Qualitymark, 2002.

LAPA C. M. F.; PEREIRA C. M. N. A.; BARROS M. P. A model for preventive maintenance planning by genetic algorithms based in cost and reliability. Reliability Engineering and System Safety 2006, 91: 233-40. cross ref

MACHADO, F.; ANDRADE, J. J. O. Emprego da confiabilidade para o estabelecimento de estratégias de manutenção na indústria metal-mecânica. XXXIII ENCONTRO NACIONAL DE ENGENHARIA DE PRODUCAO, Salvador, 2013.

MENDES, A. A. Manutenção centrada em confiabilidade: uma abordagem quantitativa. 2011. Dissertação (Mestrado em Engenharia) - Universidade Federal do Rio Grande do Sul, Brasil.

RIBEIRO, J. L. D. Apostila Confiabilidade e Manutenção. Universidade Federal do Rio Grande do Sul, 2014.

SLACK, N.; CHAMBERS, S. \& JOHNSTON, R. Administração da Produção. São Paulo: Editora Atlas, 2002.

SMITH, D. J. Reliability, maintainability and risk: practical methods for engineers. Oxford: Butterworth Heinemann, 6 ed., 2001. 335 p.

XENOS, H. G. Gerenciando a Manutenção Produtiva. Nova Lima: INDG, 2004. 


\section{Dados dos autores:}

Nome completo: Lucas Silveira Santos

Filiação institucional: Universidade Federal do Rio Grande do Sul - UFRGS

Departamento: Engenharia de Produção e Transportes

Função ou cargo ocupado: Graduando em Engenharia de Produção

Endereço completo para correspondência (bairro, cidade, estado, país e CEP): Rua Walir Zottis, 85,

Bairro Jardim Planalto, Porto Alegre - Rio Grande do Sul - Brasil, CEP 91220500

Telefones para contato: (51) 8417-3634

e-mail: silveira.santos@ufrgs.br

Nome completo: Fernanda Pinotti

Filiação institucional: Universidade Federal do Rio Grande do Sul - UFRGS

Departamento: Engenharia de Produção e Transportes

Função ou cargo ocupado: Graduando em Engenharia de Produção

Endereço completo para correspondência (bairro, cidade, estado, país e CEP): Rua Praça Carlos

Simão Arnt, 105/902, Bairro Bela Vista, Porto Alegre - Rio Grande do Sul - Brasil, CEP 90450110

Telefones para contato: (51) 9848-8030

e-mail:fernandapinotti06@gmail.com

\section{Nome completo: José Luis Duarte Ribeiro}

Filiação institucional: Universidade Federal do Rio Grande do Sul - UFRGS

Departamento: Engenharia de Produção e Transportes

Função ou cargo ocupado: Professor Associado

Endereço completo para correspondência (bairro, cidade, estado, país e CEP): Avenida Luiz Manoel Gonzaga, 915, casa 2, Bairro Petrópoilis, Porto Alegre - Rio Grande do Sul - Brasil, CEP 90470280

Telefones para contato: (51) 3308-4005

e-mail: ribeiro@producao.ufrgs.br

Nome completo: Heitor Cabral Botelho

Filiação institucional: Petrobrás

Departamento: REFAP/MI/ED

Função ou cargo ocupado: Engenheiro de Manutenção Sênior

Endereço completo para correspondência (bairro, cidade, estado, país e CEP): Avenida Pirapó, 34/401, Bairro Petrópolis, Porto Alegre - Rio Grande do Sul - Brasil, CEP 90470450 
Telefones para contato: (51) 3415-2298

e-mail: hcbotelho@petrobras.com.br

Submetido em 30-01-2015

Aceito em: 14-09-2015 